\title{
Estudio sísmico de los edificios agregados de obra de fábrica utilizando el método del espectro de capacidad
}

\section{Seismic study in masonry aggregated buildings using the capacity spectrum method}

\author{
$\underline{\text { R. Moreno-González }}^{(*)}$, J. M. Bairán ${ }^{(*)}$
}

RESUMEN

Se presenta un estudio sobre el comportamiento sísmico de un conjunto de edificios agregados de obra de fábrica situados en la ciudad de Barcelona, España. Considerando la edad que tienen estos edificios (más de 70 años) se definen dos propiedades mecánicas para la mampostería. La acción sísmica considerada se define a partir del espectro elástico de respuesta definido en el Eurocódigo 8 para la aceleración sísmica de Barcelona. Para analizar los edificios se utiliza el método del espectro de capacidad, a partir del cual se obtienen la curva de capacidad y las curvas de fragilidad para evaluar el daño sísmico esperado. Las matrices de probabilidad de daño cuantifican el daño sísmico y se obtienen a partir de las curvas de fragilidad y el punto de capacidad por demanda. Teniendo en cuenta que Barcelona se encuentra en una zona de baja sismicidad, los resultados obtenidos muestran un daño sísmico importante.

Palabras clave: Edificios agregados; mampostería; daño sísmico; curvas de capacidad; matrices de probabilidad de daño.

\section{ABSTRACT}

In this work a study about the seismic behaviour of a set of masonry aggregates buildings located in the city of Barcelona, Spain, is presented. Considering the age of these buildings (more than 70 years) two mechanical properties for the masonry were defined. The seismic action considered is defined by elastic response spectrum defined by Eurocode 8 for the seismic acceleration of Barcelona. To analyze these buildings the capacity spectrum method was used, from which the capacity curve and the fragility curves are obtained for evaluating the seismic expected damage. Damage probability matrices quantified the seismic damage and they are obtained from the fragility curves and the performance point. Considering that the city of Barcelona is located in an area of low seismicity, the results show an important seismic damage.

Keywords: Aggregated buildings; masonry; seismic damage; capacity curve; damage probability matrices.

(*) Universitat Politècnica de Catalunya, Barcelona (España).

Persona de contacto/Corresponding author: mgrosangel@ciccp.es (R. Moreno-González)

Cómo citar este artículo / Citation: Moreno-González, R., Bairán, J.M. (2014). Estudio sísmico de los edificios agregados de obra de fábrica utilizando el método del espectro de capacidad. Informes de la Construcción, 66(533): eoo1. doi: http://dx.doi.org/10.3989/ ic.11.002.

Licencia / License: Salvo indicación contraria, todos los contenidos de la edición electrónica de Informes de la Construcción se distribuyen bajo una licencia de uso y distribución Creative Commons Reconocimiento no Comercial 3.o. España (cc-by-nc). 


\section{INTRODUCCIÓN}

Los estudios de daño sísmico, a nivel estructural, tienen como finalidad evaluar la vulnerabilidad sísmica en zonas urbanas y estimar el daño probable que se puede sufrir a causa de un movimiento sísmico. La problemática de llevar a cabo este tipo de estudios de vulnerabilidad y de análisis de daño sísmico es la cantidad de información que se debe disponer y que muchas veces no se tiene.

Para evaluar el daño sísmico se requiere de alguna metodología que permita analizar, de forma fiable, la vulnerabilidad sísmica de los edificios. Existen dos grupos de metodologías que requieren de información muy variable, éstas son las específicas y las urbanas. Las metodologías específicas se basan en realizar análisis estructural estático y/o dinámico para uno o varios edificios, para ello se necesita conocer las dimensiones en planta y alzado, las secciones, las propiedades mecánicas de los materiales, por otro lado, también se necesita conocer la acción sísmica a la que se someterá el edificio (espectros de respuesta, fuerzas laterales equivalentes). Las metodologías urbanas se basan en realizar estudios a gran escala, con ellas se pueden obtener resultados que formen parte de un contexto amplio como barrios o distritos, para esto se necesita otro tipo de información como la configuración en planta y alzado, el tipo de cimentación, el estado de conservación de los elementos estructurales y no estructurales, el año de construcción, etc, pero con éstas no se puede conocer el comportamiento de la estructura.

En este trabajo se presenta un estudio para evaluar la vulnerabilidad y el daño sísmico de una tipología ampliamente existente en la ciudad de Barcelona, España. Esta tipología corresponde a los edificios de obra de fábrica de ladrillo sin armar, conocidos también como edificios de mampostería sin refuerzo. Muchos de los edificios existentes en Barcelona, específicamente en el distrito Ensanche (Eixample en catalán), no son aislados sino que forman parte de un sistema de agregados para construir las manzanas o bloques que constituyen el distrito. El objetivo principal es evaluar, para una misma tipología, el daño sísmico en un conjunto de edificios agregados que forman parte de una manzana típica de los edificios de obra de fábrica existentes en el Eixample.

Los edificios aquí analizados han sido construidos a finales del siglo XIX y a principios del siglo XX, se ubican en el distrito Eixample y representan más del 50\% de los edificios residenciales de la ciudad. Durante la última década se han llevado a cabo varios estudios (1) (2) (3) (4) sobre la evaluación de la vulnerabilidad sísmica existente en la ciudad de Barcelona y han puesto de manifiesto un daño sísmico no despreciable (5) (6) (7).

El distrito Eixample tiene 150 años, es una zona emblemática del centro de la ciudad con un importante valor histórico, arquitectónico y cultural, se considera un proyecto pionero en la evolución del urbanismo moderno. Este distrito fue construido según el diseño urbanístico del ingeniero Ildefonso Cerdá, cuyo proyecto final fue aprobado en 1860. Esta gran transformación urbanística se realizó después de ser derribadas las murallas que cercaban la ciudad (18581868) y supuso su última gran expansión. Hoy en día, este distrito destaca como la principal área de Barcelona donde se acumula una importante población, una notable actividad económica y un importante patrimonio cultural (8).
Actualmente, el municipio de Barcelona se extiende en una superficie de 9907 Ha con una población de 1,6 millones de habitantes según datos del departamento de estadísticas del Ayuntamiento de Barcelona.

El distrito Eixample está formado por unas 520 manzanas, simétricas y alineadas. En cada manzana hay un promedio de 25 edificios, las dimensiones de estas manzanas son $113 \mathrm{~m} \times$ $113 \mathrm{~m}$; en las esquinas existen chaflanes de aproximadamente unos 20m de longitud (9) (Figura 1). En general, los edificios típicos son de obra de fábrica no reforzada con forjados (losas) de viguetas de madera o viguetas metálicas, según la época, y bovedillas de hormigón o de mampostería construidas manualmente. La mayoría de estas edificaciones tienen una vista en planta rectangular y están situadas a lo largo de la calle, excepto las de esquina, situadas en los chaflanes de las calles, las cuales tienen una forma particular (pentagonal).

El sistema estructural de estos edificios está formado por paredes de carga y muestran esquemas muy repetitivos, tales como: cimentación superficial sobre zapatas corridas bajo muros, o aisladas bajo pilares, los elementos resistentes son muros de carga y elementos de sustentación en planta baja, las paredes de carga o bien secundarias se apoyan sobre un sistema de jácenas metálicas o de pórticos de hormigón, normalmente las jácenas metálicas se apoyan sobre paredes de mampostería. Las paredes alcanzan longitudes superiores a $15 \mathrm{~m}$. Estos edificios presentan una geometría rectangular y en la parte central suele existir uno o más núcleos parcialmente cerrados mediante paredes de fábrica de ladrillo de $10 \mathrm{~cm}$ de espesor, formando las cajas de escaleras o los patios de luces (Figura 2).

En las paredes existen aberturas para puertas y ventanas que dan lugar a dinteles o antepechos de anchura y altura variables. Los muros de fachadas actúan como muros portantes (paredes de carga) junto a un conjunto de paredes interiores de carga, paralelas a aquéllas. En los edificios estrechos, el forjado se forma en sentido transversal actuando las paredes de medianería (secundarias) como muros de carga. En los edificios de esquina, la distribución de las paredes es en forma trapecial, las fachadas de calle y patio actúan como paredes de carga, se dispone de una segunda pared de carga interior paralela a la fachada de calle, también se utilizan otras

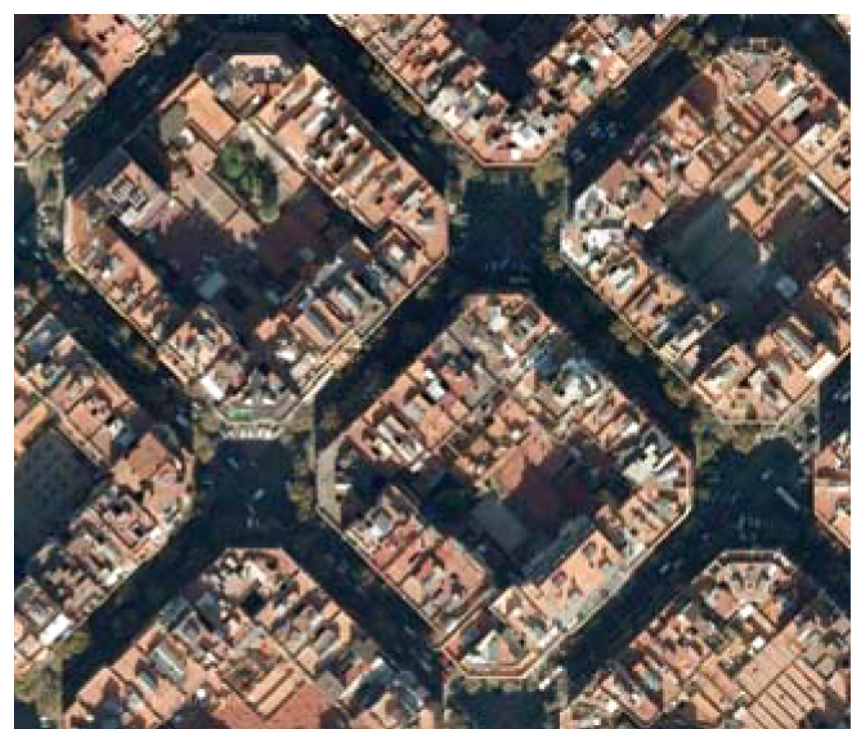

Figura 1. Vista de una manzana típica del Eixample. 


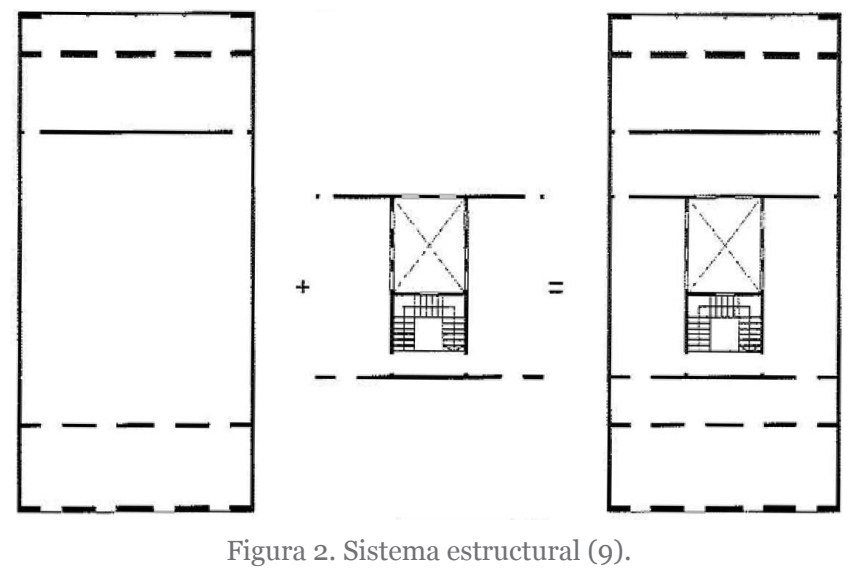

paredes interiores como elementos de carga; de esta forma existen paredes de carga en al menos tres direcciones, una dirección transversal paralela al chaflán, y dos direcciones diagonales paralelas a los lados de la manzana, separadas angularmente a $45^{\circ}$.

Los forjados son unidireccionales y presentan luces cortas. Dependiendo del año de construcción es previsible que se trate de forjados de viguetas de madera, metálicas, o de hormigón sin capa superior de compresión. Los forjados de vigueta metálica y revoltón cerámico son comunes en los techos de planta baja, apoyándose en jácenas y pilares metálicos o bien en paredes, y los forjados de vigueta de madera y revoltón cerámico son comunes en el resto de las plantas, están formados por dos hojas de rasilla y sobre éstas existen cascotes de relleno de mortero de cal y pavimento. El revoltón constituye el sistema tradicional y las bovedillas de cerámica o de mortero son rellenas de cascotes de cemento o mortero de baja resistencia.

En general, esta tipología tiene las mismas técnicas constructivas sistematizadas, debido a que se repiten constantemente, dando origen a un sistema constructivo que varía, básicamente, en la fachada exterior. Por la época en que fueron construidos no presentan ninguna consideración de diseño sísmico, lo que hace intuir que su vulnerabilidad sísmica es alta.

Finalmente, para evaluar la vulnerabilidad y el daño sísmico del conjunto de edificios agregados, el cual forma parte de una de las manzanas existentes en el Eixample de Barcelona, se utilizó una metodología del tipo "específica» basada en el método del espectro de capacidad. Para este estudio se consideró un escenario sísmico definido por la norma sísmica española NCSE-O2 (10) y el Eurocódigo 8 (11), la aceleración sísmica básica para la ciudad de Barcelona es de 0,04 g (10). Después de definir los materiales, las propiedades mecánicas y geométricas se evalúa la capacidad de los edificios con el programa TreMuri (12), obteniendo las curvas de capacidad y los espectros de capacidad bilineales, seguidamente, se definen las curvas de fragilidad y, posteriormente, se obtienen las matrices de probabilidad de daño, las cuales indican la probabilidad de que se dé un estado de daño para la acción sísmica considerada (13).

\section{MODELOS DE EDIFICIOS}

Como se dijo anteriormente, el sistema constructivo del Eixample utiliza técnicas constructivas que se sistematizan debido a que se repiten constantemente, dando origen a un sistema constructivo que varía básicamente en la fachada diferenciando los edificios de línea con los de esquina. En este apartado se definen los modelos de edificios utilizados para representar y evaluar la vulnerabilidad del sistema de edificios agregados que constituyen una de las cuatro líneas que forman o definen una manzana del Eixample de Barcelona.

Antes de estudiar el conjunto de edificios agregados primero se analizan tres modelos de edificios de forma aislada en sus dos direcciones principales, paralela y perpendicular a la calle, dirección x e y, respectivamente. Estos modelos reciben el nombre de EC, EP y EA. EC y EP son representativos de los edificios situados dentro de las hileras o líneas de edificios cuya fachada principal da a una misma calle, son de planta rectangular y no forman esquina. Por otro lado, EA tiene una planta más irregular y muestra tres secciones de fachada, dos que dan a calles distintas (calles perpendiculares) y una al chaflán que las une. EA es típico de las esquinas correspondientes a los cruces entre dos calles. La Figura 3 muestra las plantas y la numeración de las paredes de los modelos analizados. Los tres edificios son reales y se ubican en el distrito Eixample, las plantas han sido tomadas, directamente, de planos estructurales.

El edificio EC tiene en planta 12,65 $\mathrm{m} \times 27,00 \mathrm{~m}$ y en altura 22,0 $\mathrm{m}$ con un total de 7 pisos. La altura de piso en planta baja es de 4,0 m y de 3,0 m en el resto de los pisos. En planta baja las paredes de la fachada principal y trasera tienen espesores de $50 \mathrm{~cm}$ y $45 \mathrm{~cm}$ y a partir del primer piso son de $40 \mathrm{~cm}$ y $30 \mathrm{~cm}$, respectivamente. Las paredes laterales o medianeras tienen un espesor de $30 \mathrm{~cm}$ en la planta baja y de $15 \mathrm{~cm}$ en el resto de las plantas, las paredes interiores son de $15 \mathrm{~cm}$ de espesor. La edad del edificio es de 80 años.

En cuanto al edificio EP las dimensiones en planta son de $18,4 \mathrm{~m} \times 23,7 \mathrm{~m}$, lo que hace un área total de $436,08 \mathrm{~m}^{2}$, la altura total es de $24,4 \mathrm{~m}$, la planta baja tiene una altura de piso de 4,8 $\mathrm{m}$, el primer piso tiene 4,0 $\mathrm{m}$ y el resto de los pisos superiores tienen una altura de $3,9 \mathrm{~m}$ cada uno, con un total de 6 pisos. Las paredes son de $15 \mathrm{~cm}$ de espesor y las paredes de fachadas de $30 \mathrm{~cm}$. En las dos primeras plantas existen pilares y jácenas metálicas, los cuales soportan el peso de las paredes superiores. El año de construcción es 1882-1886.

El edificio EA tiene 8 pisos con una altura total de 22,4 $\mathrm{m}$ distribuidos de la siguiente manera: planta baja $=3,1 \mathrm{~m}$, primer piso $=2,4 \mathrm{~m}$ y 2,8 $\mathrm{m}$ el resto de los pisos. El área en planta es de $557 \mathrm{~m}^{2}$ y su perímetro de $94,30 \mathrm{~m}$. Las paredes presentan una mayor diversidad de formas geométricas, están dispuestas de forma paralela las unas con las otras para crear un sistema resistente. El muro de fachada de calle tiene un espesor de $40 \mathrm{~cm}$ y el de la fachada trasera de $30 \mathrm{~cm}$, las paredes interiores de carga son de $15 \mathrm{~cm}$ de espesor. El sistema resistente interior en las dos primeras plantas está formado de pilares y jácenas metálicas. La edad del edificio es de 75 años.

El forjado considerado es un forjado de losas de viguetas de madera (Figura 4) cubiertas de revoltón y con una base de baldosa doblada encajada en el interior para sostenerlo y cubierto de mortero de cal.

Para el análisis del sistema de agregados se han combinado 7 edificios de los ya definidos, formado por 2 edificios tipo EA, 3 tipo ECy 2 tipo EP.A partir de un estudio global simplificado el conjunto de agregados se ha analizado en dirección $x$ mediante la siguiente combinación: $\mathrm{EA}_{1}+\mathrm{EC}_{1}+\mathrm{EP}_{1}+\mathrm{EC}_{2}+\mathrm{EP}_{2}+\mathrm{EC}_{3}+\mathrm{EA}_{2}$ 

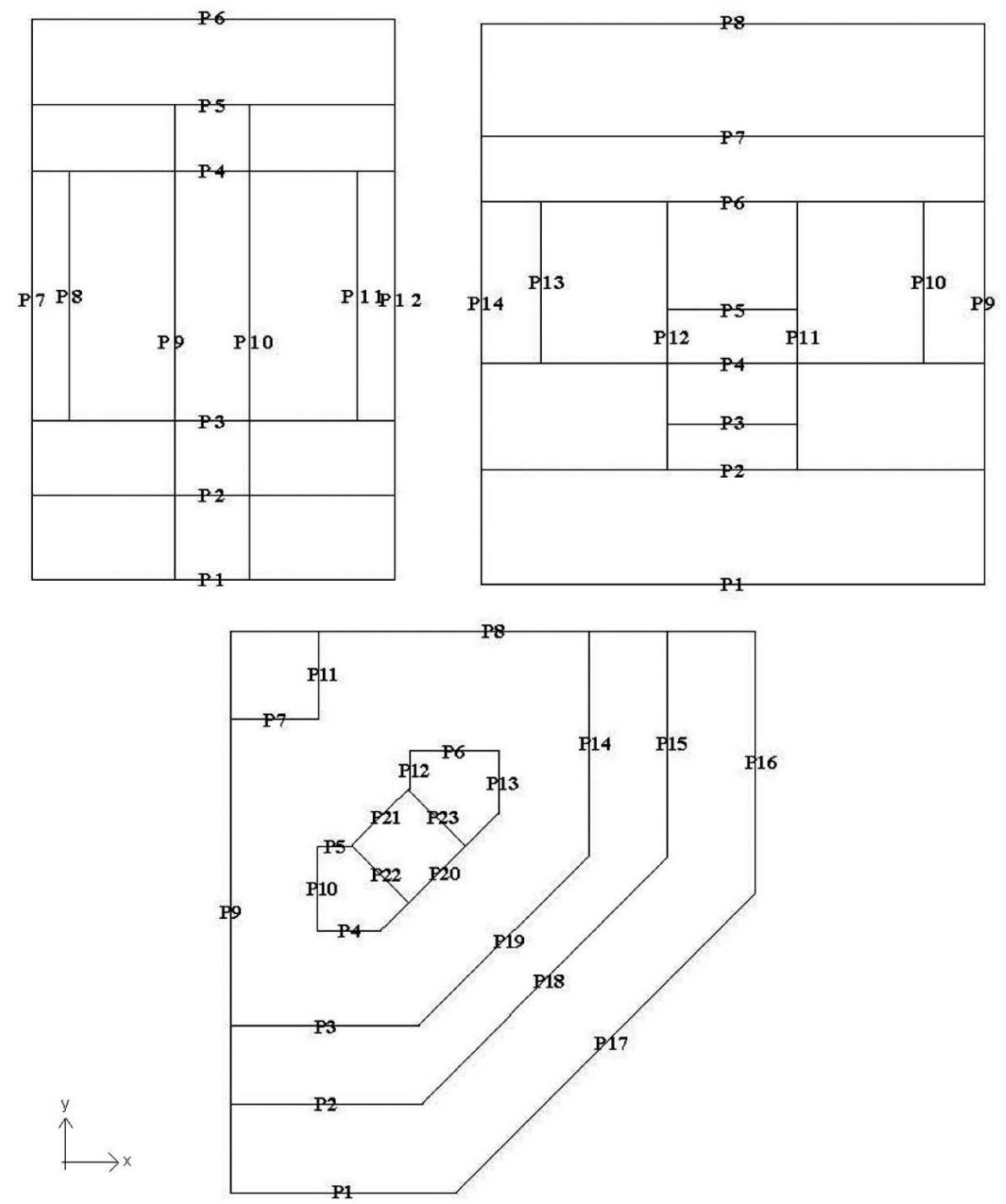

Figura 3. Plantas de los edificios analizados. EC (arriba izquierda), EP (arriba derecha) y EA (abajo).

(Figura 5). El análisis también se efectuó para otras combinaciones de 7 edificios obteniéndose resultados muy parecidos, aquí sólo se presentan los resultados correspondientes a ésta combinación. Para referenciar a esta línea de edificios agregados se les asignó el nombre de AgregadosX. Para llevar a cabo el análisis se utilizó un programa tridimensional de análisis no lineal para estructuras de mampostería llamado TreMuri (12), el cual permite realizar análisis estático y dinámico no lineal de edificios de mampostería en $3 \mathrm{D}$.

\subsection{Propiedades mecánicas}

Para evaluar el comportamiento de los edificios es necesario conocer sus propiedades mecánicas. Se desconoce la existencia de estudios rigurosos que permitan definir con exactitud las propiedades mecánicas de estos materiales. En base a las especificaciones técnicas al uso, a estudios patológicos realizados en algunos edificios, a las características constructivas y a los materiales utilizados se realizó un volumen importante de ensayos numéricos con el programa TreMuri (12) para caracterizar los parámetros mecánicos que requiere la definición de los macroelementos (14).

En base a toda esta información se optó por efectuar los análisis para dos tipos de obra de fábrica que dan lugar a dos con- figuraciones, las cuales se van a calificar como configuración típica (ct) y configuración fuerte ( $c f)$. Durante el desarrollo de este trabajo, se utiliza la configuración típica para ilustrar la construcción detallada de los espectros de capacidad y de las curvas de fragilidad, siendo ésta la más probable para los edificios de la ciudad. Al final del documento se facilita, mediante las matrices de probabilidad de daño, información sobre la influencia que tienen las variaciones de las propiedades mecánicas resistentes de los paneles de mampostería en el daño esperado.

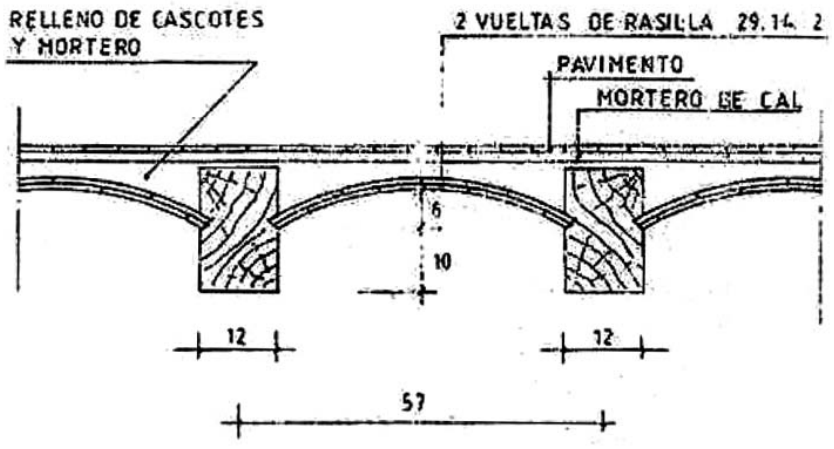

Figura 4. Forjado de madera (tomado de los planos). 


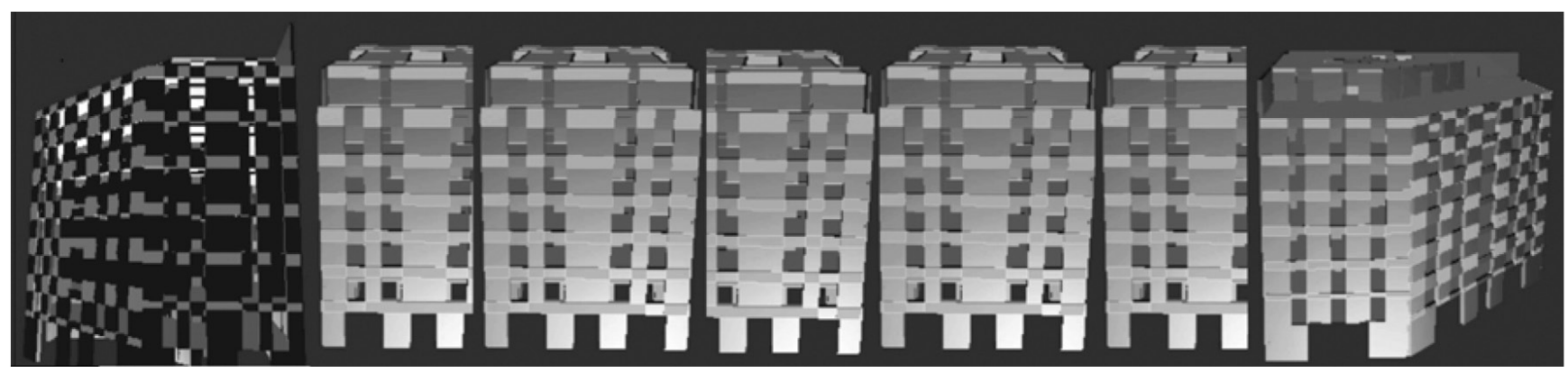

Figura 5. Configuración del modelo de agregados: $\mathrm{EA}_{1}, \mathrm{EC}_{1}, \mathrm{EP}_{1}, \mathrm{EC}_{2}, \mathrm{EP}_{2}, \mathrm{EC}_{3}, \mathrm{EA}_{2}$.

La Tabla 1 contiene las propiedades características de los macroelementos utilizados para modelar las paredes y los muros de obra de fábrica. En la misma tabla se dan los parámetros para las configuraciones constructivas aquí definidas como típica (ct) y fuerte ( $c f$ ), así como también, las características de los forjados, las propiedades de los pilares y de las jácenas metálicas existentes en algunos edificios.

\section{DESCRIPCIÓN DEL PROGRAMA TREMURI}

El programa TreMuri ha sido desarrollado en la Università degli Studi di Genova, Italia (12) es un programa tridimensional de análisis no lineal para estructuras de fábrica de ladrillo. El programa representa los paneles de mampostería mediante un modelo no lineal de macroelementos propuesto por Gambarotta y Lagomarsino (14). El TreMuri está proyectado para un análisis incremental no lineal con control de fuerza o desplazamiento, análisis estático, análisis modal y análisis dinámico con excitación sísmica en la base de la estructura, bidimensional o tridimensional.

El modelo de daño utilizado en el programa para ensamblar el modelo de la pared (macromodelo) tiene en cuenta el efec-

Tabla 1.

Propiedades mecánicas de los elementos.

\begin{tabular}{|l|r|c|}
\hline \multicolumn{3}{|c|}{ Paredes de Mampostería } \\
\hline \multicolumn{1}{|c|}{ ct } & cf \\
\hline Módulo elástico, $E$ & $1800 \mathrm{~N} / \mathrm{mm}^{2}$ & $3600 \mathrm{~N} / \mathrm{mm}^{2}$ \\
\hline Módulo a cortante, $G$ & $300 \mathrm{~N} / \mathrm{mm}^{2}$ & $600 \mathrm{~N} / \mathrm{mm}^{2}$ \\
\hline Resistencia a cortante, $\tau_{k}$ & $0,12 \mathrm{~N} / \mathrm{mm}^{2}$ & $0,12 \mathrm{~N} / \mathrm{mm}^{2}$ \\
\hline Resistencia a compresión, $f_{m k}$ & $4,0 \mathrm{~N} / \mathrm{mm}^{2}$ & $4,0 \mathrm{~N} / \mathrm{mm}^{2}$ \\
\hline Peso específico, $\gamma$ & $18 \mathrm{kN} / \mathrm{m}^{3}$ \\
\hline $\begin{array}{l}\text { Parámetro de ablandamiento para } \\
\text { pilares, } \beta_{p}\end{array}$ & 0,20 \\
\hline $\begin{array}{l}\text { Parámetro de ablandamiento para } \\
\text { vigas, } \beta_{d}\end{array}$ & 0,05 \\
\hline \multicolumn{2}{|c|}{ Forjado de Madera } \\
\hline Módulo elástico, $E_{1}$ & $4000 \mathrm{~N} / \mathrm{mm}^{2}$ \\
\hline Módulo elástico, $E_{2}$ & $40 \mathrm{~N} / \mathrm{mm}^{2}$ \\
\hline Módulo a cortante, $G$ & $100 \mathrm{~N} / \mathrm{mm}^{2}$ \\
\hline Carga, $q$ & $5 \mathrm{kN} / \mathrm{m}^{2}$ \\
\hline \multicolumn{3}{|c|}{ Perfiles Metálicos } \\
\hline \multicolumn{2}{|c|}{ Pilares } & Vigas \\
\hline Tipo de perfiles & $\mathrm{HEB}-200$ & $\mathrm{IPN}-360$ \\
\hline Módulo elástico, $E_{s}$ & $2 ., \mathrm{E} 5 \mathrm{~N} / \mathrm{mm}^{2}$ & $2,1 \mathrm{E} 5 \mathrm{~N} / \mathrm{mm}^{2}$ \\
\hline Área, $A$ & $0,00781 \mathrm{~m}^{2}$ & $0,0097 \mathrm{~m}^{2}$ \\
\hline Momento de inercia, $I_{y}$ & $56,97 \mathrm{E}-6 \mathrm{~m}^{4}$ & $19,61 \mathrm{E}-5 \mathrm{~m}^{4}$ \\
\hline Peso específico, $\gamma$ & $78,50 \mathrm{kN} / \mathrm{m}^{3}$ \\
\hline
\end{tabular}

to de la resistencia a compresión limitada de la mampostería, especialmente en el mecanismo del vuelco (15); además, incluye los efectos de agrietamiento por medio de una ley constitutiva no lineal con deterioro de la rigidez en compresión (16). A parte de sus características geométricas, el macroelemento es definido por seis parámetros: el módulo de cortante, la rigidez axial, la resistencia a cortante de la mampostería, un coeficiente adimensional que controla la deformación inelástica, un coeficiente global de fricción y un factor que controla la fase del ablandamiento (16).

Los modelos numéricos que incorpora el programa han sido calibrados cuidadosamente, de forma que el programa es capaz de reproducir los mecanismos de daño y de disipación de energía observados en casos reales. En efecto, TreMuri es capaz de describir y simular el comportamiento no lineal en el plano de los paneles de mampostería y permite estimar el daño que este tipo de edificios sufre cuando se ven sometidos a sismos. La Figura 6 muestra, a manera de ejemplo, una vista $3 \mathrm{D}$ y una de las paredes de uno de los edificios aquí modelados con dicho programa.

\section{DEMANDA SÍSMICA}

La acción sísmica se considera en términos espectrales de acuerdo a la norma sísmica española NCSE-02 (10), donde la aceleración básica de Barcelona es de 0,04 g, la cual corresponde a un período de retorno de 475 años. Para las formas de los espectros elásticos de respuesta con $5 \%$ de amortiguamiento se han utilizado los definidos en el Eurocódigo 8 (11), donde se consideran hasta 5 tipos de suelo: A, B, C, D y E, que van desde suelos duros (tipo A) a suelos blandos (tipo D);

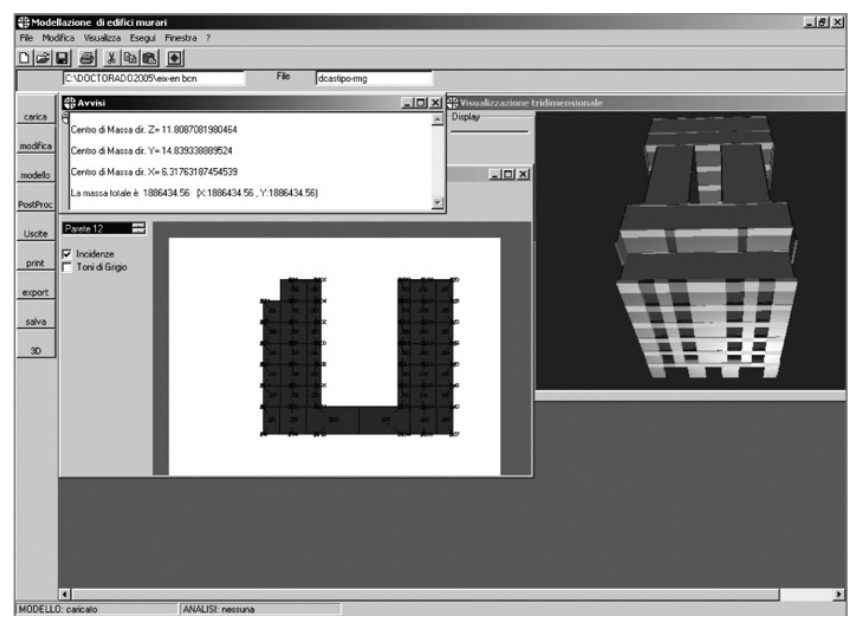

Figura 6. Vista 3D del edificio EC modelado con el programa TreMuri. 
los suelos tipo E corresponden a una configuración de suelos con un nivel aluvial de tipo $\mathrm{C}$ ó $\mathrm{D}$ sobre un material más duro (tipo A).

La Tabla 2 muestra los parámetros que definen los espectros de respuesta utilizados; donde, $S$ es un parámetro del suelo, $k_{1}$ $\mathrm{y} k_{2}$ son los exponentes que definen la forma del espectro para un período de vibración mayor que $T_{C}$ y $T_{D}$, respectivamente. $T_{B}$ y $T_{C}$ son los límites del tramo de aceleración espectral constante y $T_{D}$ define el comienzo del tramo de desplazamiento constante (11). La Figura 7 muestra los espectros elásticos de respuesta utilizados para un amortiguamiento del $5 \%$.

Tabla 2.

Parámetros que definen los espectros de respuesta.

\begin{tabular}{|c|c|c|c|c|c|c|}
\hline Tipo de suelo & $\boldsymbol{S}$ & $\boldsymbol{k}_{\boldsymbol{1}}$ & $\boldsymbol{k}_{\boldsymbol{2}}$ & $\boldsymbol{T}_{\boldsymbol{B}}(\mathbf{s})$ & $\boldsymbol{T}_{\boldsymbol{C}}(\mathbf{s})$ & $\boldsymbol{T}_{\boldsymbol{D}}(\mathbf{s})$ \\
\hline $\mathrm{A}$ & $1, \mathrm{OO}$ & $1, \mathrm{O}$ & $2, \mathrm{O}$ & 0,15 & 0,40 & $2, \mathrm{O}$ \\
\hline $\mathrm{B}$ & $1,2 \mathrm{O}$ & $1, \mathrm{O}$ & $2, \mathrm{O}$ & 0,15 & 0,50 & $2, \mathrm{O}$ \\
\hline $\mathrm{C}$ & 1,15 & $1, \mathrm{O}$ & $2, \mathrm{O}$ & 0,20 & 0,60 & $2, \mathrm{O}$ \\
\hline $\mathrm{D}$ & 1,35 & $1, \mathrm{O}$ & $2, \mathrm{O}$ & 0,20 & 0,80 & $2, \mathrm{O}$ \\
\hline $\mathrm{E}$ & $1,4 \mathrm{O}$ & $1, \mathrm{O}$ & $2, \mathrm{O}$ & 0,15 & $0,5 \mathrm{O}$ & $2, \mathrm{O}$ \\
\hline
\end{tabular}

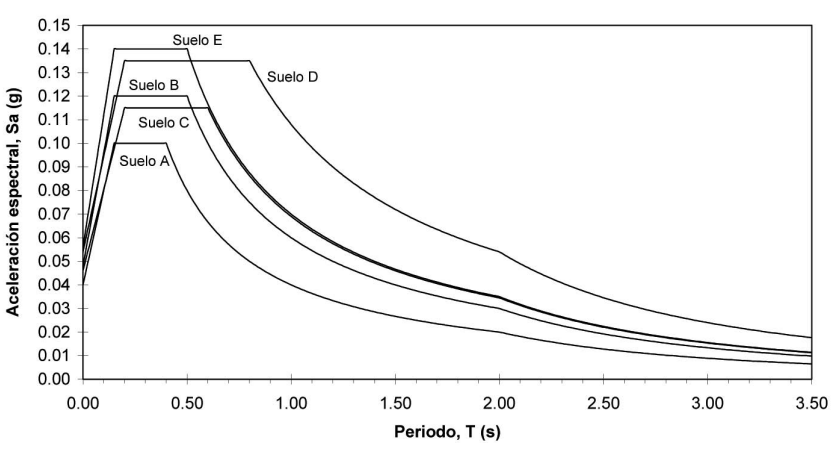

Figura 7. Espectros elásticos de respuesta para Barcelona.

\section{ANÁLISIS MODAL}

Para cada modelo estructural se obtienen sus propiedades modales. La Tabla 3 muestra, para ambas configuraciones utilizadas (ct: típica y $c f$ : fuerte), las características modales correspondientes a los primeros modos de vibración. $T$ es el período natural de vibración en segundos, $U x$ indica el desplazamiento en la dirección paralela a la calle y $U y$ el desplazamiento en la dirección perpendicular a la calle.

Tabla 3.

Valores modales para los edificios EC, EP y EA.

\begin{tabular}{|c|c|c|c|}
\hline \multirow{2}{*}{ Edificio } & \multirow{2}{*}{ Modos } & \multicolumn{2}{|c|}{ T (s) } \\
\cline { 2 - 4 } & & (ct) & (cf) \\
\hline \multirow{2}{*}{ EC } & Modo 1 $(U x)$ & 0,85 & 0,59 \\
\cline { 2 - 4 } & $\operatorname{Modo} 3(U y)$ & 0,56 & 0,40 \\
\hline \multirow{2}{*}{ EP } & $\operatorname{Modo} 1(U x)$ & 1,24 & 0,95 \\
\cline { 2 - 4 } & Modo 3 $(U y)$ & 0,80 & 0,57 \\
\hline \multirow{2}{*}{ EA } & Modo 1 $(U y)$ & 0,83 & 0,60 \\
\cline { 2 - 4 } & Modo 2 $(U x)$ & 0,81 & 0,57 \\
\hline
\end{tabular}

Para el análisis de cada edificio de forma aislada (independientemente el uno del otro) se elige el modo de vibración que produce un desplazamiento longitudinal en dirección $y$, es decir, se utilizan las propiedades modales correspondientes al modo 3 para los edificios EC y EP, y al modo 1 para el edificio EA.
Para el estudio del modelo AgregadosX los edificios se analizan en dirección $x$. Se considera que los edificios situados en una misma línea de manzana tienen propiedades mecánicas, alturas y sistemas constructivos similares, por lo que para AgregadosX el período en el sistema equivalente de un grado de libertad es $\mathrm{T}^{*}=0,818$ segundos para la configuración típica y para la configuración fuerte $\mathrm{T}^{*}=0,606 \mathrm{~s}$.

\section{VULNERABILIDAD SÍSMICA: CURVAS DE CAPACIDAD Y CURVAS DE FRAGILIDAD}

La vulnerabilidad de un edificio se puede definir como la capacidad que tiene la estructura de soportar acciones sísmicas. La metodología utilizada se basa en el método del espectro de capacidad donde, después de un análisis modal, se realiza un análisis estático no lineal (empuje progresivo) para obtener las curvas de capacidad, éstas curvas son transformadas a espectros de capacidad para su posterior comparación con el espectro de demanda.

Con la finalidad de tener parámetros objetivos y cuantificables para evaluar la capacidad y fragilidad de cada estructura, el espectro de capacidad se representa por medio de una curva de capacidad bilineal simplificada, definida por dos puntos, el punto de capacidad de cedencia (Dy, $A y)$ y el punto de capacidad última $(D u, A u)$, donde las áreas sobre y bajo la curva de capacidad original deben ser iguales (Figura 8). El punto de capacidad de cedencia representa el desplazamiento en el que la respuesta del edificio empieza a ser no lineal y el punto de capacidad última representa el desplazamiento en el que el sistema estructural ha alcanzado el mecanismo de colapso o su máxima capacidad. Dy es el desplazamiento espectral de cedencia y $A y$ representa la ordenada de $D y, D u$ es el desplazamiento espectral último y $A u$ es la ordenada de $D u$ (17). Esta representación bilineal es necesaria para definir los umbrales de los estados de daño y, posteriormente, construir las curvas de fragilidad.

Ante una acción sísmica definida y dada una respuesta estructural, las curvas de fragilidad definen la probabilidad de alcanzar o exceder un estado de daño límite específico. En la metodología HAZUS (18), las curvas de fragilidad siguen una distribución de probabilidad lognormal y se definen con respecto a una variable que caracteriza la acción sísmica, en este caso, el desplazamiento espectral $(S d)$. Así pues, las curvas de fragilidad se definen aquí como [1].

$$
P\left[E D \geq E D_{i}\right]=\Phi\left[\frac{1}{\beta_{E D}} \cdot \ln \left(\frac{S d}{S d_{E D}}\right)\right]
$$

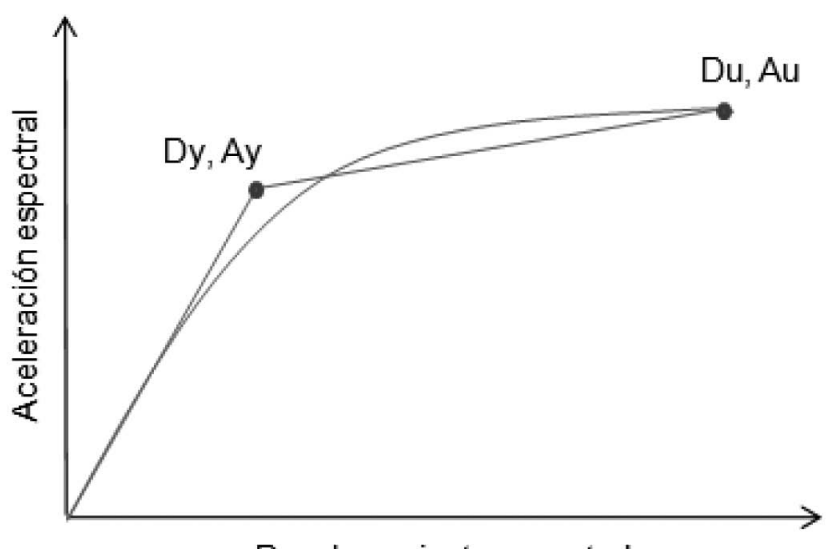

Desplazamiento espectral

Figura 8. Definición de la curva de capacidad bilineal 
donde $\overline{S d}_{E D}$ es el desplazamiento espectral medio de cada estado de daño para el cual la probabilidad de excedencia es del $50 \%, \beta_{E D}$ es la variabilidad asociada al estado de daño, $\Phi$ es la función de distribución normal típica acumulada, $S d$ es el desplazamiento espectral, $E D_{i}$ indica el estado de daño y viene definido como: $i=1$ para el estado de daño leve, $i=2$ para moderado, $i=3$ para severo y $i=4$ para el estado de daño de colapso (13). Estas curvas se representan gráficamente con la probabilidad de alcanzar o exceder un estado de daño, $P\left(E D>E D_{i}\right)$, en las ordenadas y con el desplazamiento espectral $(S d)$ en las abscisas (19).

Los umbrales de los estados de daño se definen con los desplazamientos espectrales medios, los cuales se obtienen de forma simplificada a partir del espectro de capacidad bilineal. La Tabla 4 y la Figura 9 muestran la definición de los umbrales de los estados de daño en función de los desplazamientos de cedencia y último (6). Así pues, las curvas de fragilidad están relacionadas con las curvas de capacidad mediante el espectro de capacidad bilineal.

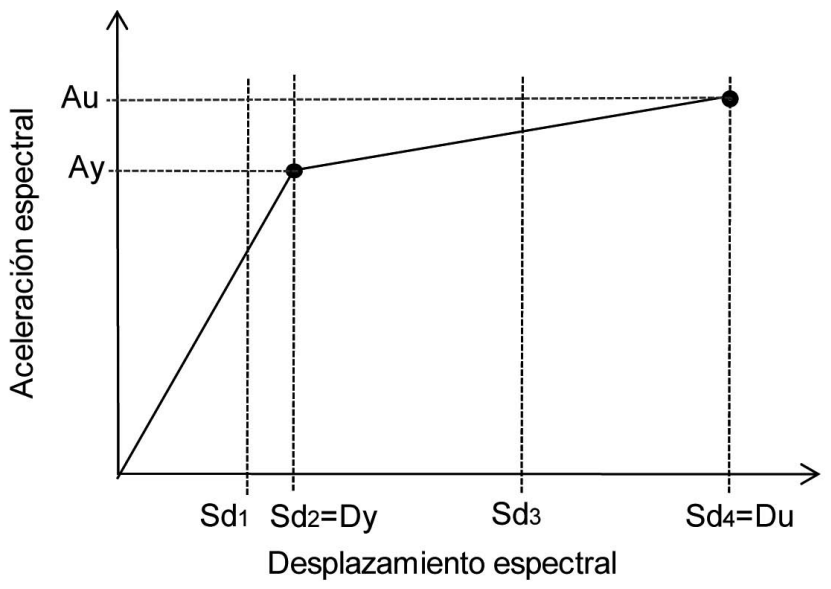

Figura 9. Definición gráfica de los umbrales de los estados de daño.

Tabla 4 .

Definición de los umbrales de los estados de daño.

\begin{tabular}{|l|l|}
\hline Umbrales de daño & \multicolumn{1}{|c|}{ Definición } \\
\hline Leve & $\overline{S d}_{1}=0,7 \cdot D y$ \\
\hline Moderado & $\overline{S d}_{2}=D y$ \\
\hline Severo & $\overline{S d}_{3}=D y+0,25 \cdot(D u-D y)$ \\
\hline Colapso & $\overline{S d}_{4}=D u$ \\
\hline
\end{tabular}

La estimación de la desviación típica no es un problema trivial, en la metodología HAZUS (18) se definen para distintas tipologías y se basan en ensayos numéricos y en la opinión de expertos. Para la tipología aquí estudiada, los valores de $\beta_{E D}$ utilizados son: $\beta_{1}=0,99 ; \beta_{2}=0,97 ; \beta_{3}=0,90 ; \beta_{4}=0,88$ (13) (18).
A continuación se presentan las curvas de capacidad obtenidas a partir del programa TreMuri (12), el cual permite estimar adecuadamente el desplazamiento de colapso, ya que se puede observar los daños en los paneles de mampostería y su evolución, seguidamente se presentan las curvas de fragilidad de cada uno de los edificios analizados.

\subsection{Curvas de capacidad}

En este apartado se presentan las curvas de capacidad obtenidas a partir de un análisis estático no lineal (empuje progresivo) donde el patrón de fuerzas horizontales corresponde con el modo de vibración elegido.

Para los edificios EC, EP y EA se obtienen las curvas de capacidad para el modo de vibración que produce el desplazamiento Uy. Para evaluar el conjunto de edificios AgregadosX se repitió, para cada uno de los modelos, el mismo análisis anterior pero en la dirección paralela a la calle, es decir, se llevo a cabo un análisis de empuje incremental teniendo en cuenta el modo de vibración correspondiente a $U x$. Partiendo de un sistema equivalente de un grado de libertad y considerando que los edificios que conforman el sistema de agregados presentan un forjado rígido en su plano, alturas similares y un mismo sistema constructivo, se obtiene el espectro de capacidad correspondiente a AgregadosX. Estas curvas de capacidad se transforman a espectro de capacidad bilineal para su posterior utilización en la obtención de las curvas de fragilidad.

La Tabla 5 muestra los valores que definen los espectros de capacidad bilineal para los edificios EC, EP y EA, así como también se muestran los valores que definen el espectro de capacidad bilineal para el conjunto de edificios AgregadosX.

Las Figuras 10, 11, 12 y 13 muestran los espectros de capacidad bilineales obtenidos con la configuración típica para los edificios EC, EP, EA y para el sistema AgregadosX, respectivamente. En estas gráficas también se muestran representados los umbrales de los estados de daño.

\subsection{Curvas de fragilidad}

Las curvas de fragilidad para cada modelo de edificio se obtienen a partir de los espectros de capacidad bilineales (Tabla 5) donde se definen los desplazamientos espectrales medios para cada umbral del estado de daño. En las Figuras 10, 11, 12 y 13 se encuentran representados estos estados de daño sobre los espectros de capacidad bilineales. La Tabla 6 muestra, para ambas configuraciones mecánicas (ct y cf), los parámetros que definen las curvas de fragilidad correspondientes a EC, EP, EA y AgregadosX. Las Figuras 14, 15, 16 y 17 muestran las respectivas curvas de fragilidad de los edificios EC, EP, EA y AgregadosX para la configuración típica.

Tabla 5.

Parámetros que definen la curva de capacidad bilineal.

\begin{tabular}{|c|c|c|c|c|c|c|c|c|}
\hline \multirow{3}{*}{ Modelos } & \multicolumn{4}{|c|}{ Configuración típica } & \multicolumn{4}{|c|}{ Configuración fuerte } \\
\hline & \multicolumn{2}{|c|}{$\begin{array}{c}\text { Capacidad } \\
\text { de cedencia }\end{array}$} & \multicolumn{2}{|c|}{$\begin{array}{c}\text { Capacidad } \\
\text { última }\end{array}$} & \multicolumn{2}{|c|}{$\begin{array}{c}\text { Capacidad } \\
\text { de cedencia }\end{array}$} & \multicolumn{2}{|c|}{$\begin{array}{c}\text { Capacidad } \\
\text { última }\end{array}$} \\
\hline & $\begin{array}{l}\text { Dy } \\
(\mathbf{m})\end{array}$ & $\begin{array}{l}\text { Ay } \\
\text { (g) }\end{array}$ & $\begin{array}{l}\mathrm{Du} \\
(\mathrm{m})\end{array}$ & $\begin{array}{l}\text { Au } \\
\text { (g) }\end{array}$ & $\begin{array}{l}\text { Dy } \\
(\mathbf{m})\end{array}$ & $\begin{array}{l}\text { Ay } \\
\text { (g) }\end{array}$ & $\begin{array}{l}\text { Du } \\
(\mathbf{m})\end{array}$ & $\begin{array}{l}\text { Au } \\
(\mathrm{g})\end{array}$ \\
\hline $\mathrm{EC}$ & 0,015 & 0,19 & 0,071 & 0,20 & 0,008 & 0,20 & 0,041 & 0,19 \\
\hline EP & 0,025 & 0,12 & 0,17 & 0,10 & 0,013 & 0,12 & 0,092 & 0,11 \\
\hline EA & 0,011 & 0,12 & 0,051 & 0,12 & 0,007 & 0,12 & 0,037 & 0,12 \\
\hline Agregados X & 0,013 & 0,077 & 0,040 & 0,091 & 0,008 & 0,087 & 0,040 & 0,11 \\
\hline
\end{tabular}


Tabla 6.

Desplazamiento espectral medio para cada umbral de daño.

\begin{tabular}{|c|c|c|c|c|c|c|c|c|}
\hline \multirow{2}{*}{ Modelo } & \multicolumn{2}{|c|}{$\overline{S D}_{1}(\mathrm{~cm})$} & \multicolumn{2}{|c|}{$\overline{S D}_{2}(\mathrm{~cm})$} & \multicolumn{2}{|c|}{$\overline{S D}_{3}(\mathrm{~cm})$} & \multicolumn{2}{|c|}{$\overline{S D}_{4}(\mathrm{~cm})$} \\
\hline & ct & $\boldsymbol{c f}$ & ct & $\boldsymbol{c f}$ & ct & $\boldsymbol{c f}$ & ct & $\boldsymbol{c} f$ \\
\hline & 1,07 & 60 & 1,53 & 80 & 2,93 & ( & 7,12 & 4,10 \\
\hline & & & 2,5 & & , & & 16,76 & 9,2 \\
\hline & 0,77 & & & & 21 & & 5,09 & $3,7 c$ \\
\hline gregadosX & 0,91 & 0,60 & 1,30 & 0,80 & 1,97 & 1,60 & 4,00 & 4,0 \\
\hline
\end{tabular}

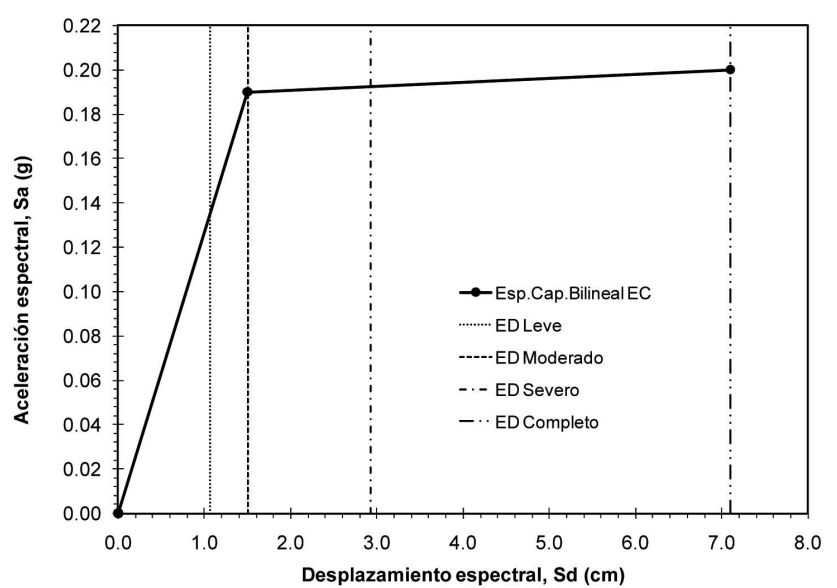

Figura 10. Espectro de capacidad y umbrales de los estados de daño para EC.

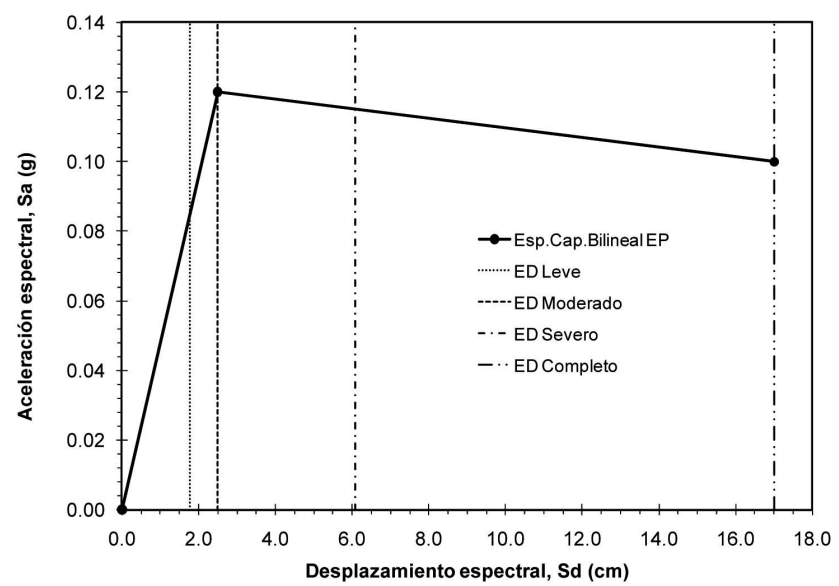

Figura 11. Espectro de capacidad y umbrales de los estados de daño para EP.

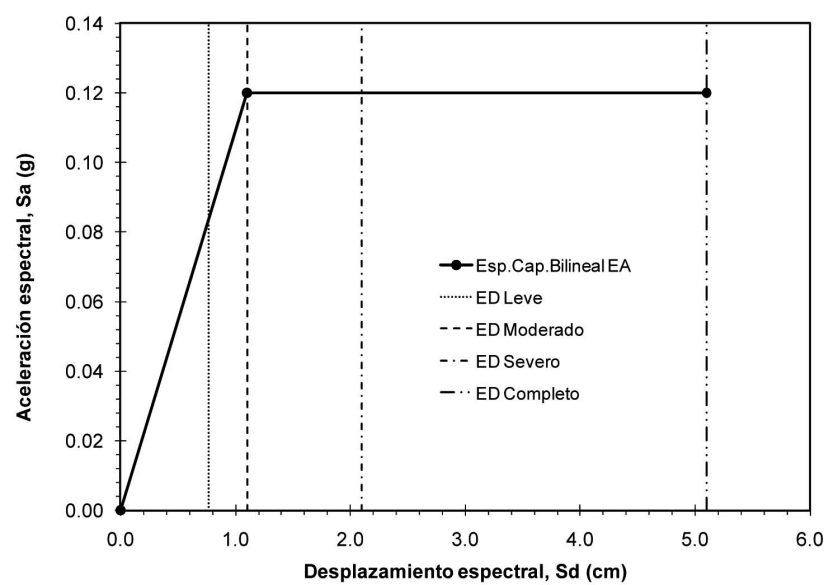

Figura 12. Espectro de capacidad y umbrales de los estados de daño para EA.

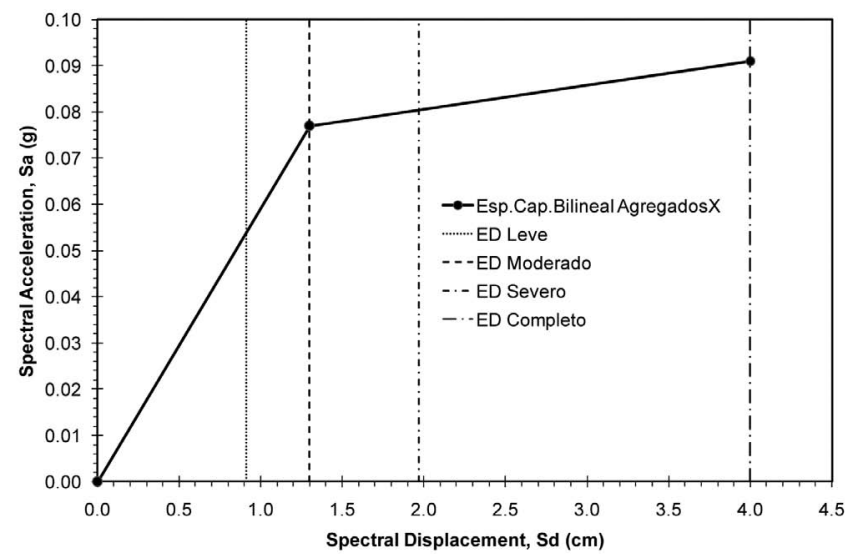

Figura 13. Espectro de capacidad y umbrales de los estados de daño para AgregadosX.

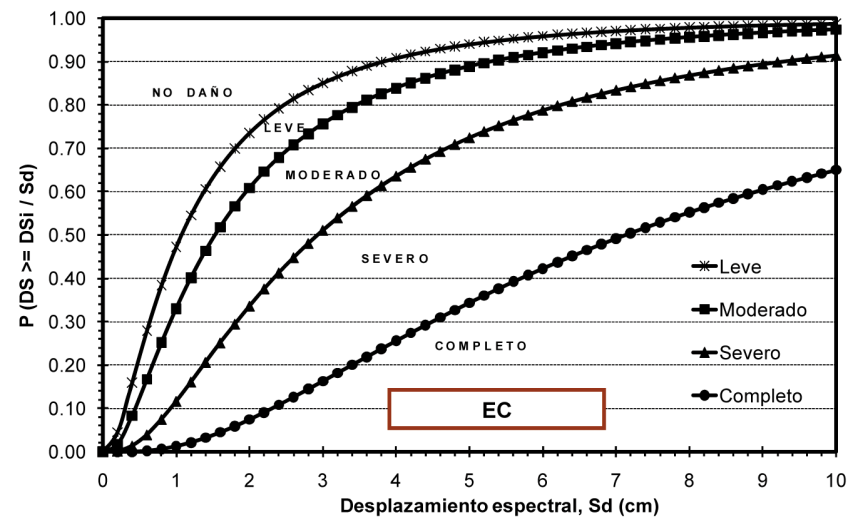

Figura 14. Curvas de fragilidad para el edificio EC.

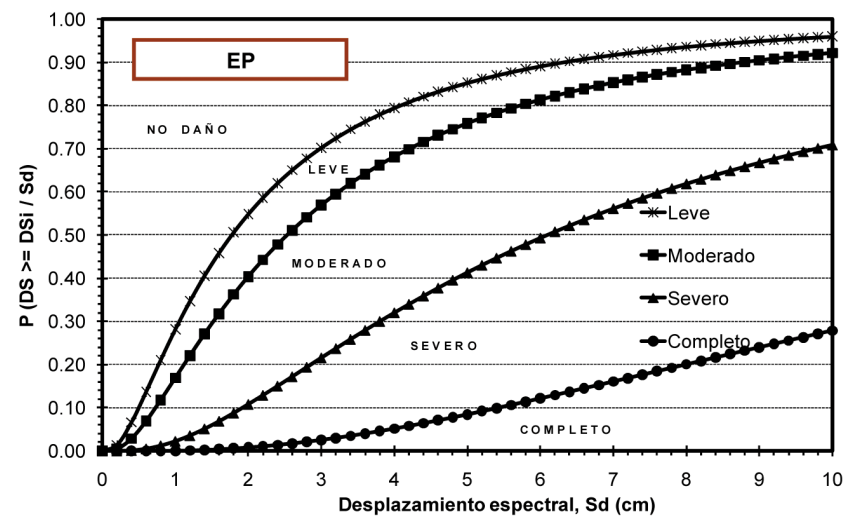

Figura 15. Curvas de fragilidad para el edificio EP.

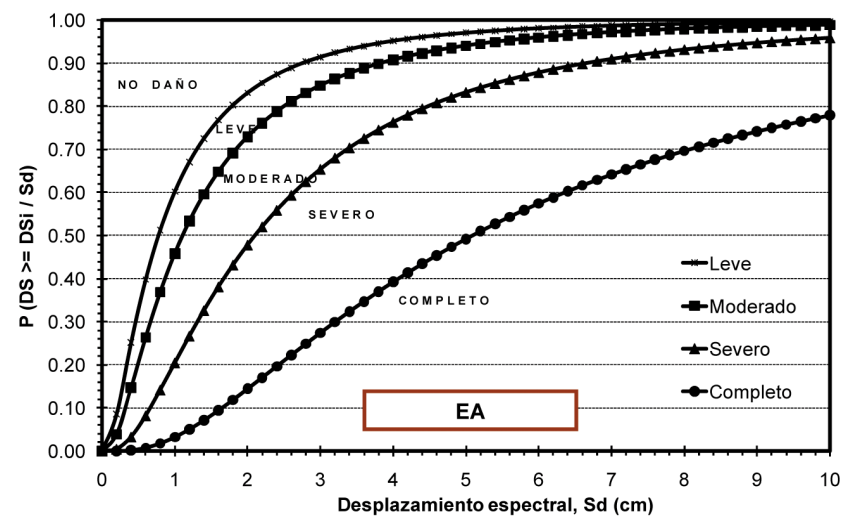

Figura 16. Curvas de fragilidad para el edificio EA. 


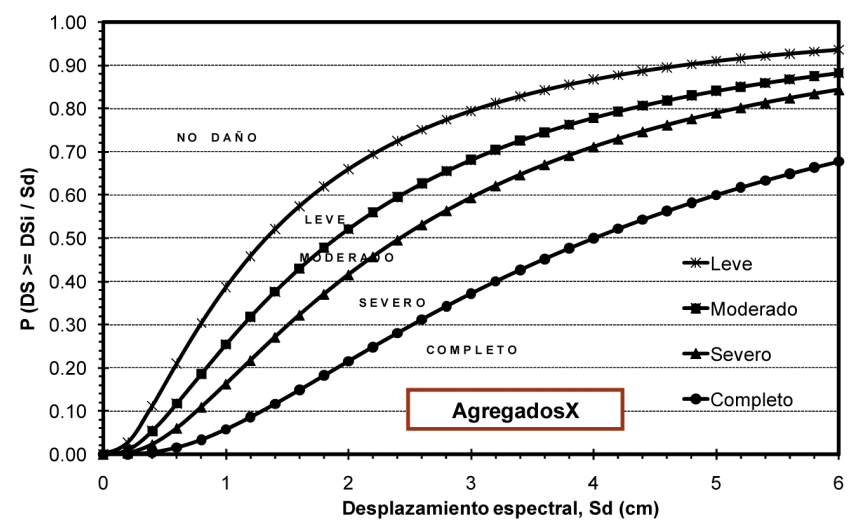

Figura 17. Curvas de fragilidad para AgregadosX.

Después de obtener las curvas de fragilidad se pueden construir las matrices de probabilidad de daño y calcular, por medio de un indicador de daño, el daño global esperado en cada estructura.

\section{EVALUACIÓN DEL DAÑO}

En este apartado, para cada escenario sísmico y para cada modelo, se definen las matrices de probabilidad de daño, las cuales se obtienen a partir de las curvas de fragilidad y del punto de capacidad por demanda. Para esto, es necesario entrar en las curvas de fragilidad con el punto de capacidad por demanda y leer las probabilidades correspondientes para cada estado de daño (19). El punto de capacidad por demanda $\left(S d_{P D}\right)$ es la máxima respuesta sísmica de un edificio cuando se encuentra ante un evento sísmico y se obtiene en el punto de intersección del espectro de capacidad con el espectro de demanda.

El espectro de demanda es la representación de la acción sísmica y se basa en el espectro de respuesta de diseño de la zona de estudio, con $5 \%$ de amortiguamiento, reducido para niveles mayores de amortiguamiento efectivo.

Existen varios métodos o procedimientos para estimar la respuesta sísmica de una estructura, en este trabajo se utilizó el procedimiento indicado en el Eurocódigo 8 (11), el cual se halla perfectamente detallado en el Anexo $B$ de dicho código. Además, se comprobó que los resultados obtenidos con éste método apenas difieren de los que se obtienen usando el procedimiento $A$ sugerido en el ATC-40 (17).

El parámetro ponderado de daño medio utilizado para cuantificar el daño sísmico se define como [2]:

$$
d_{M}=\sum_{i=0}^{i=4} E D_{i} \cdot P\left(E D_{i}\right)
$$

donde $i$ corresponde a cada estado de daño considerado y $P(E D)$ es la probabilidad de ocurrencia del estado de daño $i$ dado un desplazamiento espectral. El índice $i$ toma valores de o, 1, 2, 3 y 4 para el estado de daño: no daño, leve, moderado, severo y colapso, respectivamente (19).

Para los cinco estados de daño considerados los intervalos de variación de $d_{M}$ se definen como, no daño: o-o,5, leve: 0,5-1,5; moderado: 1,5-2,5, severo: $2,5^{-3}, 5$ y colapso: $3,5^{-}$ 4,0 (19). Este parámetro de daño medio $\left(d_{M}\right)$ puede ser útil para representar escenarios de riesgo sísmico en un área determinada.

\subsection{Respuesta sísmica}

Para determinar la respuesta sísmica se han tenido en cuenta los cinco tipos de suelos definidos en el Apartado 4 y los dos tipos de materiales utilizados en el análisis. Para cada edificio, la Tabla 7 muestra los puntos de capacidad por demanda obtenidos para el escenario sísmico considerado.

\subsection{Matrices de probabilidad de daño}

Finalmente, para cada escenario sísmico y para cada edificio se obtienen las matrices de probabilidad de daño. Entrando en las curvas de fragilidad con el punto de capacidad por demanda se obtienen las probabilidades correspondientes a cada estado de daño, por lo tanto, la probabilidad de daño se define como [3]:

$$
P\left[E D_{i} / S d_{P D}\right]=P\left(E D_{i} / S d_{P D}\right)-P\left(E D_{i+1} / S d_{P D}\right)
$$

donde, $P\left[E D_{i} / S d_{P D}\right]$ es la probabilidad de excedencia del estado de daño $E D_{i}$ para la respuesta máxima estructural, definida por el punto $S d_{P D}$.

Para la aceleración de Barcelona (o,04 g) y para las dos configuraciones analizadas, la Tabla 8 muestra la variación de las matrices de probabilidad de daño para cada edificio localizado en diferentes emplazamientos.

\section{7•3. Daño medio esperado}

El grado de daño medio $\left(d_{M}\right)$ indica el estado de daño con una mayor probabilidad de ocurrencia. Para cada edificio, para el escenario sísmico considerado y para las configuraciones típica y fuerte la Tabla 9 proporciona los valores de daño medio obtenidos a partir de las matrices de probabilidad de daño. Este parámetro da una idea global del estado de daño más probable y será utilizado para comentar los resultados.

Tabla 7.

Puntos de capacidad por demanda $S d_{P D}(\mathrm{~cm})$.

\begin{tabular}{|c|c|c|c|c|c|c|c|c|c|c|}
\hline \multirow{2}{*}{ Modelos } & \multicolumn{2}{|c|}{ Suelo A } & \multicolumn{2}{c|}{ Suelo B } & \multicolumn{2}{c|}{ Suelo C } & \multicolumn{2}{c|}{ Suelo D } & \multicolumn{2}{c|}{ Suelo E } \\
\cline { 2 - 12 } & ct & cf & ct & $\boldsymbol{c f}$ & ct & $\boldsymbol{c f}$ & ct & cf & ct & cf \\
\hline EC & 0,56 & 0,40 & 0,84 & 0,75 & 1,03 & 1,03 & 2,15 & 2,15 & 0,98 & 0,87 \\
\hline EP & 0,93 & 0,67 & 1,39 & 1,00 & 1,60 & 1,15 & 2,50 & 2,15 & 1,62 & 1,17 \\
\hline EA & 0,61 & 0,47 & 0,91 & 0,75 & 1,05 & 1,03 & 2,15 & 2,15 & 1,07 & 0,87 \\
\hline AgregadosX & 0,90 & 0,60 & 1,35 & 0,90 & 1,55 & 1,04 & 2,43 & 2,15 & 1,57 & 1,05 \\
\hline
\end{tabular}


Tabla 8.

Matrices de probabilidad de daño.

\begin{tabular}{|c|c|c|c|c|c|c|c|c|c|c|}
\hline & \multicolumn{2}{|c|}{ No Daño } & \multicolumn{2}{|c|}{ Leve } & \multicolumn{2}{|c|}{ Moderado } & \multicolumn{2}{|c|}{ Severo } & \multicolumn{2}{|c|}{ Colapso } \\
\hline & ct & cf & ct & cf & ct & cf & ct & $\mathrm{cf}$ & ct & $\boldsymbol{c f}$ \\
\hline & \multicolumn{10}{|c|}{ Suelo A } \\
\hline $\mathrm{EC}$ & 0,74 & 0,64 & 0,11 & 0,12 & 0,12 & 0,18 & 0,03 & 0,06 & 0,00 & 0,00 \\
\hline $\mathrm{EP}$ & 0,75 & 0,64 & 0,10 & 0,13 & 0,13 & 0,20 & 0,02 & 0,04 & 0,00 & 0,00 \\
\hline EA & 0,60 & 0,50 & 0,13 & 0,15 & 0,19 & 0,24 & 0,07 & 0,10 & 0,01 & 0,01 \\
\hline \multirow[t]{2}{*}{ AgregadosX } & 0,66 & 0,48 & 0,12 & 0,14 & 0,09 & 0,24 & 0,09 & 0,12 & 0,04 & 0,02 \\
\hline & \multicolumn{10}{|c|}{ Suelo B } \\
\hline $\mathrm{EC}$ & 0,60 & 0,39 & 0,13 & 0,15 & 0,19 & 0,27 & 0,07 & 0,17 & 0,01 & 0,02 \\
\hline EP & 0,60 & 0,47 & 0,14 & 0,15 & 0,21 & 0,29 & 0,05 & 0,09 & 0,00 & 0,00 \\
\hline EA & 0,43 & 0,33 & 0,15 & 0,13 & 0,24 & 0,31 & 0,16 & 0,20 & 0,02 & 0,03 \\
\hline \multirow[t]{2}{*}{ AgregadosX } & 0,50 & 0,32 & 0,14 & 0,14 & 0,10 & 0,28 & 0,15 & 0,22 & 0,11 & 0,04 \\
\hline & \multicolumn{10}{|c|}{ Suelo C } \\
\hline $\mathrm{EC}$ & 0,52 & 0,27 & 0,14 & 0,13 & 0,22 & 0,30 & 0,11 & 0,24 & 0,01 & 0,06 \\
\hline $\mathrm{EP}$ & 0,54 & 0,42 & 0,16 & 0,12 & 0,23 & 0,34 & 0,07 & 0,12 & 0,00 & 0,01 \\
\hline EA & 0,38 & 0,22 & 0,15 & 0,32 & 0,25 & 0,34 & 0,18 & 0,12 & 0,04 & 0,01 \\
\hline \multirow[t]{2}{*}{ AgregadosX } & 0,44 & 0,27 & 0,14 & 0,12 & 0,11 & 0,30 & 0,18 & 0,25 & 0,13 & 0,06 \\
\hline & \multicolumn{10}{|c|}{ Suelo D } \\
\hline $\mathrm{EC}$ & 0,24 & 0,09 & 0,13 & 0,07 & 0,27 & 0,22 & 0,28 & 0,40 & 0,08 & 0,22 \\
\hline EP & 0,36 & 0,20 & 0,14 & 0,12 & 0,34 & 0,36 & 0,14 & 0,27 & 0,02 & 0,05 \\
\hline EA & 0,15 & 0,06 & 0,10 & 0,06 & 0,24 & 0,20 & 0,35 & 0,41 & 0,16 & 0,27 \\
\hline \multirow[t]{2}{*}{ AgregadosX } & 0,27 & 0,09 & 0,13 & 0,07 & 0,10 & 0,21 & 0,22 & 0,39 & 0,28 & 0,24 \\
\hline & \multicolumn{10}{|c|}{ Suelo E } \\
\hline $\mathrm{EC}$ & 0,54 & 0,32 & 0,14 & 0,15 & 0,21 & 0,29 & 0,10 & 0,20 & 0,01 & 0,04 \\
\hline EP & 0,54 & 0,41 & 0,14 & 0,14 & 0,25 & 0,33 & 0,06 & 0,11 & 0,01 & 0,01 \\
\hline EA & 0,37 & 0,27 & 0,14 & 0,13 & 0,26 & 0,31 & 0,19 & 0,24 & 0,04 & 0,05 \\
\hline AgregadosX & 0,43 & 0,27 & 0,15 & 0,12 & 0,11 & 0,30 & 0,17 & 0,25 & 0,14 & 0,06 \\
\hline
\end{tabular}

Tabla 9.

Parámetro de daño medio $\left(d_{M}\right)$.

\begin{tabular}{|c|c|c|c|c|c|c|c|c|c|c|}
\hline \multirow{2}{*}{ Modelos } & \multicolumn{2}{|c|}{ Suelo A } & \multicolumn{2}{|c|}{ Suelo B } & \multicolumn{2}{|c|}{ Suelo C } & \multicolumn{2}{|c|}{ Suelo D } & \multicolumn{2}{|c|}{ Suelo E } \\
\hline & ct & $\boldsymbol{c f}$ & ct & $\boldsymbol{c f}$ & ct & $\boldsymbol{c f}$ & ct & $\boldsymbol{c f}$ & ct & $\boldsymbol{c f}$ \\
\hline $\mathrm{EC}$ & 0,44 & 0,66 & 0,76 & 1,28 & 0,95 & 1,69 & 1,83 & 2,59 & 0,90 & 1,49 \\
\hline $\mathrm{EP}$ & 0,42 & 0,65 & 0,71 & 1,00 & 0,83 & 1,17 & 1,32 & 1,85 & 0,86 & 1,17 \\
\hline EA & 0,76 & 0,97 & 1,19 & 1,47 & 1,35 & 1,37 & 2,27 & 2,77 & 1,39 & 1,67 \\
\hline AgregadosX & 0,73 & 1,06 & 1,23 & 1,52 & 1,42 & 1,71 & 2,11 & 2,62 & 1,44 & 1,71 \\
\hline
\end{tabular}

\section{DISCUSIONES Y CONCLUSIONES}

Los valores del factor de daño medio para el suelo E son similares a los valores obtenidos para suelos tipo C, Tabla 9, por lo que la discusión de los resultados obtenidos se va a enfocar a los suelos tipo A, B, C y D que van de mejor (tipo A) a peor calidad (tipo D).

Con la Tabla 9 se observa como para todos los edificios y para el conjunto AgregadosX el daño aumenta al disminuir la calidad del suelo. El aumento del daño esperado es espectacular para el edificio EA, que pasa de un estado de daño leve (o,760,97 ), cuando se halla en suelo duro (tipo A), a un estado de daño moderado a severo $(2,27-2,77)$, cuando se halla en suelos blandos (tipo D).

De los tres edificios analizados de forma aislada, EA es el que muestra el peor comportamiento sísmico y EP el mejor. Ello confirma los beneficios de la regularidad geométrica y de distribución de muros de los edificios que queda patente también en todos los códigos sismorresistentes. Además, la irregularidad geométrica dificulta la disposición adecuada de los elementos estructurales resistentes, lo que debilita aún más el edificio. Con los resultados obtenidos, parece que el conjunto AgregadosX hereda la falta de resistencia sísmica de los dos edificios laterales (EA). La geometría global de AgregadosX no contribuye a mejorar su comportamiento sísmico que, en los suelos A, B y C, es peor pero comparable al del edificio EA, y para suelos tipo D es también comparable pero mejor que el del edificio EA. Con todo hay que tener en cuenta que, la consideración de edificios aislados a edificios que forman parte de un conjunto de agregados, en general, subestima el daño esperado.

Los resultados muestran con claridad que los edificios a pesar de tener un mayor módulo elástico (caso fuerte) presentan un índice de daño mayor, esto se debe a la capacidad de disipación de energía que tiene la estructura en el momento de estar sometida ante un evento sísmico, es decir, al obtener la respuesta sísmica máxima de la estructura (punto de capacidad por demanda) el edificio que presenta mayor módulo elástico reduce más el espectro de demanda que aquel con un menor módulo elástico; por lo tanto, el amortiguamiento efectivo para reducir el espectro de demanda es mayor para 
los edificios evaluados con las características de configuración fuerte que para aquellos evaluados con las características de configuración típica dadas en la Tabla 1.

Se pueden considerar como intervalos máximo y mínimo de los daños los resultados obtenidos, representados por las matrices de probabilidad de daño (Tabla 8) y los factores de daño medio (Tabla 9). Por ejemplo, en suelos tipo D el edificio EP pasa de un grado de daño leve $(1,32)$ a moderado $(1,85)$, el edificio EC pasa de 1,83-moderado a 2,59-severo y el edificio EA de 2,27-moderado a 2,77-severo, claramente se observa un aumento de un grado en el estado de daño más probable.

Si se considera que la amplificación del suelo blando incrementa en un grado la intensidad macrosísmica básica, entonces para una intensidad VII, muchos edificios tipo EA sufren daños severos y muchos sufren colapso, por lo que se debería incluir este tipo de edificios dentro de la clase más vulnerable (clase de vulnerabilidad A) de las cinco clases de vulnerabilidad previstas en la escala EMS-98 (20). Por otra parte, el edificio EP situado en suelo tipo A, sufre un grado de daño medio que varía entre 0,42-0,65 y las probabilidades de los estados de daño son del orden del 10-13\% para el estado de daño leve y del 13-20\% para el estado de daño moderado; así pues, para una intensidad VI, varios edificios sufren daños moderados. Teniendo en cuenta la descripción del grado de daño VI y que las probabilidades del 10\% y del 13\% se hallan en la frontera entre las cantidades consideradas como pocos y muchos en la escala EMS-98 (20), los edificios tipo EP se encuentran dentro de la clase de vulnerabilidad B.

En conclusión, el daño esperado es alto teniendo en cuenta el escenario sísmico considerado $\left(\mathrm{a}_{\mathrm{b}}=0,04 \mathrm{~g}\right)$ definido en la norma NCSE-O2 (10), el cual corresponde a una intensidad básica VI en la escala macrosísmica europea EMS-98 (20). Por lo tanto, se concluye que los edificios de obra de fábrica de ladrillo muestran una alta vulnerabilidad para la aceleración sísmica considerada y pueden clasificarse entre la clase A y la clase B de las clases de vulnerabilidad tipificadas en la escala EMS-98 (20).

\section{AGRADECIMIENTOS}

Este trabajo estuvo parcialmente financiado por una beca Marie Curie Fellowship durante una estancia realizada en la Università degli Studi di Genova y en ROSE School (Italia). Los autores están agradecidos con el Ing. Vicente Alegre y con la empresa COTCA por la información suministrada. Además, la primera autora agradece al Dr. Andrea Penna, al Prof. Sergio Lagomarsino y a todo su equipo de trabajo por las aportaciones realizadas.

\section{REFERENCIAS}

(1) Yépez, F. (1996). Metodología para la evaluación de la vulnerabilidad y riesgo sísmico de estructuras aplicando técnicas de simulación (Tesis Doctoral). Universidad Politécnica de Cataluña, Barcelona, España.

(2) Mañà, F. (1997). Vulnerabilitat sísmica dels edificis de la ciutat de Barcelona, 98 pp. Insititut de Tecnología de la Construcció de Catalunya. Barcelona, España.

(3) Moreno, R., Bonett, R., Barbat, A., Pujades, L., Penna, A. y Lagomarsino, S. (2003). Evaluación de la vulnerabilidad sísmica de estructuras de mampostería no reforzada. Aplicación a un edificio de la zona del Eixample de Barcelona, España. Revista Internacional de Ingeniería de Estructuras, 8(2): 91-120.

(4) Moreno, R., Bairán, J., Pujades, L., Aparicio, A. y Barbat, A. (2004). Evaluación probabilista del comportamiento sísmico de edificios porticados de hormigón armado. Hormigón y Acero, 231: 125-136.

(5) Mena, U. (2002). Evaluación del riesgo sísmico en edificios en zonas urbanas. (Tesis Doctoral). Universidad Politécnica de Cataluña, Barcelona, España.

(6) Barbat, A.H., Pujades, L.G., Lantada, N., Moreno, R. (2006). Performance of buildings under earthquake in Barcelona, Spain. Computer-Aided Civil and Infrastructure Engineering, 21(8): 573-593. Erratum (2010). 25(3): 226. doi: http://dx.doi.org/10.1111/j.1467-8667.2010.0066o.x.

(7) Lantada, N. (2007). Evaluación del riesgo sísmico mediante métodos avanzados y técnicas GIS. Aplicación a la ciudad de Barcelona (Tesis Doctoral). Universidad Politécnica de Cataluña, Barcelona, España.

(8) García Espuche, A. (1990). El quadrat d'or. centre de la Barcelona modernista. La formació d'un espai urbà privilegiat. Olimpiada cultural Barcelona'92. Lunwerg Editores, 315 pp.

(9) Paricio, A. (2001). Secrects d'un sistema constructiu: l'Eixample. Universidad Politécnica de Cataluña. Ediciones UPC, Barcelona.

(10) NCSE-02: Normativa «Norma de Construcción Sismorresistente: Parte General y Edificación», Real Decreto 997/2002, Boletín Oficial del Estado: 244, Madrid, (2002).

(11) EC-8: Eurocode 8 (2004). Design of structures for earthquake resistance - Part 1: General rules, seismic actions and rules for buildings. Comité Européen de Normalisation. EN 1998-1:2004, CEN Brussels, December, 229 pp.

(12) Galasco, A., Lagomarsino, S. y Penna, A. (2002). TreMuri Program: Seismic Analyzer of 3D Masonry Buildings. Technical Report, Universitat degli Studi di Genova, Italia.

(13) Moreno-González, R. y Bairán, J.M. (2010). Análisis del comportamiento sísmico de los edificios de obra de fábrica, típicos del distrito Eixample de Barcelona. Informes de la Construcción, 63(524): 21-32, doi: http://dx.doi.org/10.3989/ ic.10.045.

(14) Gambarotta, L., Lagomarsino, S. (1997). Damage model for the seismic response of brick masonry shear walls. Part II: the continuum model and its applications. Earthquake Engineering and Structural Dynamics, 26(4): 441-462, doi: http://dx.doi.org/10.1002/(SICI)1096-9845(199704)26:4<441::AID-EQE651>3.0.CO;2-0.

(15) Penna, A. (2002). A macro-element procedure for the non-linear dynamic analysis of masonry buildings (Tesis Doctoral). Politecnico di Milano, Italia. 
(16) Galasco, A., Lagomarsino, S., Penna, A., Resemini, S. (2004). Non-linear seismic analysis of masonry buildings. 13th World Conference on Earthquake Engineering, paper 843, Vancouver, Canadá.

(17) ATC-40 (1996). Seismic Evaluation and Retrofit of Concrete Buildings. Applied Technology Council, Report: SSC 96-01, Volume 1, Seismic Safety Commission, Redwood City, California.

(18) HAZUS 99-SR2 (2002). HAZUS Technical Manual. Federal Emergency Management Agency, FEMA \& National Institute of Building Sciences, NIBS, Washington D.C., Vol. 1, 2, 3.

(19) Moreno, R. (2006). Evaluación del riesgo sísmico en edificios mediante análisis estático no lineal: Aplicación a diversos escenarios sísmicos de Barcelona (Tesis Doctoral). Universidad Politécnica de Cataluña, Barcelona, España.

(20) Grünthal, G. (editor). (1998). European Macroseismic Scale 1998. Centre Européen de Géodynamique et Séismologie, Cahiers du Centre Européen de Géodynamique et de Séismologie, Volume 15, Luxemburg. 